\title{
The mimetic finite difference method for elliptic and parabolic problems with a staggered discretization of diffusion coefficient
}

\author{
Konstantin Lipnikov*, Gianmarco Manzini*, \\ J. David Moulton*, Mikhail Shashkov ${ }^{\dagger}$
}

October 24, 2015

\begin{abstract}
Numerical schemes for nonlinear parabolic equations based on the harmonic averaging of cell-centered diffusion coefficients break down when some of these coefficients go to zero or their ratio grows. To tackle this problem, we propose new mimetic finite difference schemes that use a staggered discretization of the diffusion coefficient. The primary mimetic operator approximates $\operatorname{div}(k \cdot)$; the derived (dual) mimetic operator approximates $-\nabla(\cdot)$. The new mimetic schemes preserve symmetry and positivedefiniteness of the continuum problem which allows us to use algebraic solvers with optimal complexity. We perform detailed numerical analysis of the new schemes for linear elliptic problems and a specially designed linear parabolic problem that has solution dynamics typical for nonlinear problems. We show that the new schemes are competitive with the state-of-the-art schemes for steady-state problems but provide much more accurate solution dynamics for the transient problem.
\end{abstract}

\section{Background}

Modeling of complex geophysical subsurface and surface flows or nonlinear heat conduction in cold materials, requires numerical schemes that remain accurate under extreme conditions where the diffusion coefficient is not only discontinuous but may also become degenerate. A typical equation is $\partial p / \partial t+\operatorname{div} \mathbf{v}=0$ where $\mathbf{v}=-k(p) \nabla p$. The model has applications in heat diffusion [4] and moisture transport in porous media [16]. Numerical schemes for this equation that use harmonic averaging of cell-centered diffusion coefficients break down when some of these coefficients go to zero or their ratio grows, see Fig. 1.

\footnotetext{
${ }^{1}$ Applied Mathematics and Plasma Physics Group, Theoretical Division, Los Alamos National Laboratory, \{lipnikov,manzini,moulton\}@lanl.gov

${ }^{2}$ XCP-4 Group, Computational Physics Division, Los Alamos National Laboratory, shashkov@lanl.gov
} 
The list of compatible discretization methods that have the same problem includes the standard mimetic finite difference (MFD) [3, 13], hybrid finite volume (FV) [7, 8], mixed FV [5], discrete duality FV [11, 12], mixed finite element (FE) [15], and virtual element [9] methods.

Replacement of the harmonic averaging with arithmetic averaging leads to an accurate solution in one dimension, see the right panel in Fig. 1. In multiple dimensions, a similar strategy leads to a non-symmetric scheme, unless a two-point flux approximation (TPFA) formula is employed. While the formula is known to be inaccurate on general polygonal and polyhedral meshes, lack of symmetry implies only conditional positive definiteness of the resulting matrices.
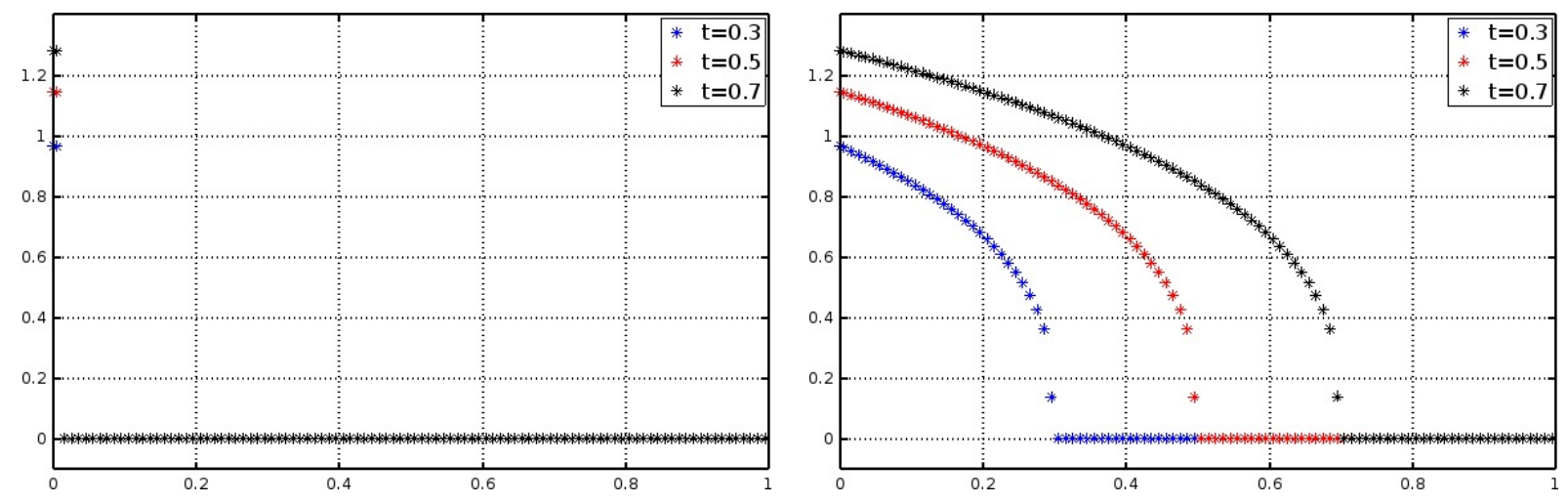

Figure 1: Solution snapshots in one dimension for $k(p)=p^{3}, p(x, 0)=10^{-3}$, and $p(0, t)=$ $\sqrt[3]{3 t}$. The panels show solutions of a conventional finite difference scheme with the harmonic (left) and arithmetic (right) averaging of the cell-centered diffusion coefficients. Accurate solution approximation is on the right panel.

The families of mimetic schemes developed here use a different approach based on a new discrete calculus. In the true mimetic spirit they guarantee symmetry and positive definiteness of the resulting discrete systems on arbitrary polygonal and polyhedral meshes. These properties typically lead to better performance of algebraic solvers, such as multigrid solvers. The proposed schemes allow us to use two diffusion coefficients (more generally, two approximations) on each mesh face. In porous media applications, the nonlinear coefficient is a continuous function of $p$ but due to its strong nonlinearity different flow regimes (infiltration, drainage, and capillary rise) require different strategies for calculating facebased diffusion coefficients that are optimal in some sense. There is strong evidence that flexibility in approximating face-based diffusion coefficients will be demanded for solving nonlinear problems.

It is pertinent to mention a related expanded mixed FE scheme proposed in [1]. This scheme is limited to finite element meshes, use two velocity unknowns $\mathbf{u}=-\nabla p$ and $\mathbf{v}=k \mathbf{u}$, and employ only cell-centered diffusion coefficients. This scheme avoids inversion of $k$ which happens in the conventional mixed FE schemes. In contrast to the proposed schemes, upwinding of the diffusion coefficients on mesh faces, which is the well established practise for solving nonlinear problems in subsurface flows, cannot be incorporated into the expanded mixed FE scheme. 
Analysis of the new mimetic schemes for linear problems is necessary before moving to nonlinear problems. We demonstrate with numerical experiments that the new schemes are competitive with the existing schemes for steady-state elliptic problems but provide much more accurate solution for a specially designed linear parabolic problem that has solution dynamics typical for nonlinear problems.

The MFD method preserves or mimics essential mathematical and physical properties of underlying PDEs. We refer the reader to the review paper [13] and book [2] for the detailed description of the MFD method. The review paper summarizes almost all known results on Cartesian and curvilinear meshes for various PDEs including the Lagrangian hydrodynamics. The book complements the paper by providing numerous examples and describing basic tools used in the convergence analysis of mimetic schemes for elliptic PDEs. For parabolic problems the essential properties include the corresponding conservation law, as well as the symmetry and positive-definiteness of the underlying differential operator.

In the mimetic framework, we discretize pairs of adjoint operators, such as the primary divergence and the derived gradient operators. Most aforementioned FV and FE methods, including the original MFD method, discretize the divergence operator $\operatorname{div}(\cdot)$ and the flux operator $-k \nabla(\cdot)$. The new mimetic schemes use a novel discrete calculus where the primary operator discretizes the combined operator $\operatorname{div}(k \cdot)$ and the derived (dual) gradient operator discretizes $-\nabla(\cdot)$. Note that the original MFD schemes use inner products in the space of fluxes that are weighted by $k^{-1}$; while the new mimetic scheme use inner products weighted by $k$. Using a one-dimensional example, we show that the new schemes can reproduce a few known methods by selecting different formulas for the diffusion coefficients on mesh faces.

The mimetic technology generates a parameterized family of schemes (even on simplicial meshes) with equivalent properties [2]. This family may contain members with superior properties such as the discrete maximum principle [10]. As an additional result, we proved that a linear reconstruction of the diffusion coefficient inside mesh cells may be incorporated in the new schemes. This is also true for the original mimetic schemes. Numerical experiments indicate that the gradient of this reconstruction does not alter the optimal convergence rate; hence, it increases the parametric space.

The paper outline is as follows. In Section 2, we present a non-standard mixed formulation of the diffusion problem. In Section 3, we develop a new approach to building mimetic schemes. The properties of the new schemes are verified through numerical experiments in Section 4. Summary of main results is given in Section 5.

\section{Mixed formulation of the diffusion problem}

In a domain $\Omega \subset \Re^{d}, d=2$ or 3 , we consider the linear parabolic equation

$$
a \frac{\partial p}{\partial t}-\operatorname{div}(k \nabla p)=b
$$

subject to initial and boundary conditions. Hereafter, $p$ is referred to as the pressure, $k=k(\mathbf{x}, t)$ is a positive, possibly discontinuous, scalar function of space and time, $b(\mathbf{x})$ is the source term, and $a(\mathbf{x}) \geq 0$. Without loss of generality, we consider homogeneous 
Dirichlet boundary condition $p=0$ on $\partial \Omega$. Other types of boundary conditions can be also embedded into the discretization technology, see Section 3.2.5.

Let us introduce a vector variable $\mathbf{u}=-\nabla p$ (referred to as the velocity) and reformulate the diffusion problem (2.1) as a system of two first-order equations:

$$
\mathbf{u}=-\nabla p \quad \text { and } \quad a \frac{\partial p}{\partial t}+\operatorname{div}(k \mathbf{u})=b .
$$

Note that only the physical velocity $k \mathbf{u}$ has continuous normal components across the discontinuity of $k$. When degrees of freedom are associated with gradients, a special numerical treatment of $k$ is required to get a convergence scheme. In addition, the above equations may not have a strong solution when $k$ is discontinuous and must be understood in a weak sense.

In this paper, we assume that $k$ is uniformly bounded from below and above, so that the original continuum problem is well posed and has a weak solution. Extension of the proposed technology to nonlinear problems with degenerate coefficients will be done in future publications.

Let $\Omega_{h}$ be a conformal partition of $\Omega$ into polygonal $(d=2)$ or polyhedral $(d=3)$ cells $c$. This mesh may contain non-convex cells. Let $h_{c}$ denote the diameter of cell $c$ and $|c|$ denote its volume. We use symbol $f$ for a mesh face and $|f|$ for its area. Hereafter, we use mainly 3D notations to describe the method with a few remarks about lower dimensions. We assume that mesh faces match discontinuity interfaces of function $k$. Under this assumption, it is possible to develop a rigorous convergence theory.

\section{Mimetic finite difference method}

Let $\mathcal{F}_{h}$ and $\mathcal{P}_{h}$ be discrete spaces (formalized later) for the primary unknowns. In the MFD method the semi-discrete equations have the same structure as that in (2.2): Find $\mathbf{u}_{h} \in \mathcal{F}_{h}$ and $p_{h} \in \mathcal{P}_{h}$ such that

$$
\mathbf{u}_{h}=-\mathcal{G} \mathcal{R} \mathcal{A D} p_{h} \quad \text { and } \quad a \frac{\partial p_{h}}{\partial t}+\mathcal{D} \mathcal{I} \mathcal{V}^{k} \mathbf{u}_{h}=b^{I}
$$

where $\mathcal{D} \mathcal{I} \mathcal{V}^{k}$ is the primary mimetic operator that approximates the continuum combined operator $\operatorname{div} k, \mathcal{G} \mathcal{R} \mathcal{A D}$ is the derived mimetic operator that approximates $\nabla$, and $b^{I} \in \mathcal{P}_{h}$ is a projection of the source term. As described later, the gradient operator is dual to the primary operator (with respect to inner products in $\mathcal{F}_{h}$ and $\mathcal{P}_{h}$ ) which is critical for proving symmetry as well as the positive definiteness of the resulting discrete systems. In numerical experiments in Section 4, we use the backward Euler time discretization. Since the time discretization is not critical for the scheme derivation, we set $a=0$ in this section.

In subsequent sections we specify discrete spaces for $p_{h}$ and $\mathbf{u}_{h}$. To simplify exposition of the main idea, we start with a 1D problem where all constructions become transparent. Based on the definition of the derived gradient operator, schemes I and II can be classified as global schemes, while scheme III is a local one. 


\subsection{Illustration of main concepts in $1 \mathrm{D}$}

Let $\Omega=(0,1)$. We consider a non-uniform mesh with $n$ cells as shown in Fig. 2. The primary velocity $u_{i}, i=1, \ldots, n+1$ and pressure $p_{i-1 / 2}, i=1, \ldots, n$, unknowns are defined at mesh nodes and cell centers, respectively. Let $\mathbf{u}_{h}$ and $p_{h}$ be vectors collecting all velocity and pressure unknowns. Finally, let $h_{i+1 / 2}$ denote the size of cell $\left(x_{i}, x_{i+1}\right)$.

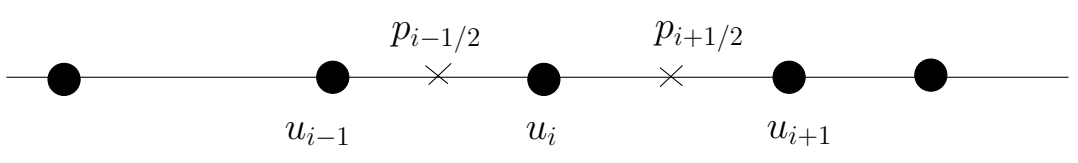

Figure 2: Degrees of freedom in the mimetic scheme. Velocity unknowns are defined at mesh nodes that are marked with solid disks. Pressure unknowns are defined at cell centers.

We choose the primary mimetic operator in each mesh cell as a straightforward discretization of the divergence theorem:

$$
\left(\mathcal{D} \mathcal{I} \mathcal{V}^{k} \mathbf{u}_{h}\right)_{i-1 / 2}=\frac{1}{h_{i-1 / 2}}\left(k_{i} u_{i}-k_{i-1} u_{i-1}\right), \quad 1 \leq i \leq n,
$$

where $k_{i}$ is some approximation of $k$ at node $\mathbf{x}_{i}$ that will be specified later. This is the essential difference with other schemes (e.g., mixed FE and FV) that approximate effectively divergence operator div.

To find the derived mimetic operator, we consider the following integration by parts formula:

$$
\int_{0}^{1} k(\nabla p) \cdot \mathbf{u} d x=-\int_{0}^{1}(\operatorname{div} k \mathbf{u}) p d x \quad \forall \mathbf{u} \in H^{1}(\Omega), p \in H_{0}^{1}(\Omega) .
$$

The formula implies that operator $\operatorname{div}(k \cdot)$ is negatively adjoint to operator $\nabla(\cdot)$ with respect to the weighted $L^{2}$ products ( $k$ is the weight). A discrete integration by parts formula mimics (3.2). Both integrals are replaced by accurate approximations that we will refer to as inner products. The approximation of the second integral is quite straightforward. Since we have only one pressure value in each mesh cell, we break the integral into integrals over mesh cells and employ the mid-point quadrature rule in each cell:

$$
\int_{0}^{1}(\operatorname{div}(k \mathbf{u})) p d x=\sum_{i=1}^{n} h_{i-1 / 2}\left(\mathcal{D} \mathcal{I} \mathcal{V}^{k} \mathbf{u}_{h}\right)_{i-1 / 2} p_{i-1 / 2}+O(h) .
$$

Dropping off the $O(h)$ term, we obtain the inner product in the space of pressure unknowns. The approximation of the first integral in (3.2) is not unique and leads to a few schemes described below.

\subsubsection{Scheme I}

In the first mimetic scheme, we approximate $k$ by a single value $\tilde{k}^{i-1 / 2}$ in each mesh cell. Hereafter, symbol tilde indicates diffusion coefficients associated with interior of mesh cells. 
To approximate the first integral in (3.2), we use the trapezoidal quadrature rule in each mesh cell which leads to the following discrete Green formula:

$$
\sum_{i=1}^{n} \tilde{k}^{i-1 / 2} \frac{h_{i-1 / 2}}{2}\left[\left(\mathcal{G} \mathcal{R} \mathcal{A D} p_{h}\right)_{i-1} u_{i-1}+\left(\mathcal{G} \mathcal{R} \mathcal{A D} p_{h}\right)_{i} u_{i}\right]=-\sum_{i=1}^{n} h_{i-1 / 2}\left(\mathcal{D} \mathcal{I} \mathcal{V}^{k} \mathbf{u}_{h}\right)_{i-1 / 2} p_{i-1 / 2}
$$

Inserting the formula for the divergence and changing the summation from cells to nodes, we obtain the explicit formula for the derived gradient operator:

$$
\left(\mathcal{G} \mathcal{R} \mathcal{A D} p_{h}\right)_{i}=-\frac{k_{i}\left(h_{i-1 / 2}+h_{i+1 / 2}\right)}{h_{i-1 / 2} \tilde{k}^{i-1 / 2}+h_{i+1 / 2} \tilde{k}^{i+1 / 2}} \frac{p_{i-1 / 2}-p_{i+1 / 2}}{\left(h_{i-1 / 2}+h_{i+1 / 2}\right) / 2}
$$

where $p_{1 / 2}=p_{N+1 / 2}=0$. When $k=1$, the coefficient in front of the discrete pressure gradient is one. Approximation of the physical velocity is

$k_{i} u_{i}=-k_{i}\left(\mathcal{G} \mathcal{R} \mathcal{A D} p_{h}\right)_{i}=\frac{k_{i}^{2}\left(h_{i-1 / 2}+h_{i+1 / 2}\right)}{h_{i-1 / 2} \tilde{k}^{i-1 / 2}+h_{i+1 / 2} \tilde{k}^{i+1 / 2}} \frac{p_{i-1 / 2}-p_{i+1 / 2}}{\left(h_{i-1 / 2}+h_{i+1 / 2}\right) / 2} \equiv T_{i}\left(p_{i-1 / 2}-p_{i+1 / 2}\right)$.

Inserting this formula in the mass balance equation and multiplying each equation by the cell volume, we obtain a system of algebraic equations for the pressure unknowns only. The matrix of this system is symmetric, positive definite, and tridiagonal. The off-diagonal entries in this matrix are given by coefficients $T_{i}$.

At this moment, $k_{i}$ is still an arbitrary approximation of the diffusion coefficient. We can recover the conventional FV scheme [6] with harmonic averaging of cell-centered diffusion coefficients for a special choice of $k_{i}$ given by

$$
k_{i}=\sqrt{k_{i}^{H} k_{i}^{A}}
$$

where

$$
k_{i}^{H}=\left(\frac{h_{i-1 / 2}}{\tilde{k}^{i-1 / 2}}+\frac{h_{i+1 / 2}}{\tilde{k}^{i+1 / 2}}\right)^{-1}\left(h_{i-1 / 2}+h_{i+1 / 2}\right), \quad k_{i}^{A}=\frac{h_{i-1 / 2} \tilde{k}^{i-1 / 2}+h_{i+1 / 2} \tilde{k}^{i+1 / 2}}{h_{i-1 / 2}+h_{i+1 / 2}} .
$$

When the above formula for the gradient is used as the basis for solving a problem with the degenerate (or close to zero) diffusion coefficient, we observe a clear difference between various selections of $k_{i}$. For instance, we show in Section 4 that the choice $k_{i}=k_{i}^{H}$ fails when $\tilde{k}^{i+1 / 2} \approx 0$. The problem with harmonic averaging was already reported in the literature, e.g. in $[17,14]$. For the scheme considered in this section, the choice $k_{i}=k_{i}^{A}$ leads to a better scheme. Later, we show how to extend this good scheme to multiple dimensions.

Often, in a computer code, only cell-centered values $\tilde{k}^{i-1 / 2}$ are available for our scheme and values $k_{i}$ are derived somehow from them. Let $k_{i}$ be the s-th order approximation of the diffusion coefficient at point $x_{i}$, where $1 \leq s \leq 2$. The truncation error analysis on a non-smooth mesh i.e. $h_{i+1 / 2}-h_{i-1 / 2} \sim O(h)$, shows three terms in the flux error:

$$
\left|(k u)\left(x_{i}\right)-k_{i} u_{i}\right| \leq C h\left(\left|k^{\prime} p^{\prime}\right|+\left|p^{\prime \prime}\right|\right)+C h^{s}+O\left(h^{2}\right),
$$


where $C$ is a positive constant independent of the mesh, solution and diffusion coefficient. If the mesh is smooth, i.e. $h_{i+1 / 2}-h_{i-1 / 2} \sim O\left(h^{2}\right)$, the truncation error estimate becomes

$$
\left|(k u)\left(x_{i}\right)-k_{i} u_{i}\right| \leq C h^{s}+O\left(h^{2}\right) .
$$

Hence, the second-order estimate of the flux requires $s=2$. On a smooth mesh, a variety of averaging and limited reconstructions algorithms with such a property is available. We show with 2D and 3D numerical experiments that a more accurate approximation of $k_{i}$ results in a much smaller discretization error.

\subsubsection{Scheme II}

Let us assume that exact or approximate nodal values $k_{i}$ are given. The second mimetic scheme is obtained when we approximate $k$ by a linear function in each mesh cell using $k_{i}$. The trapezoidal rule gives us to the following discrete integration by parts formula:

$$
\sum_{i=1}^{n} \frac{h_{i-1 / 2}}{2}\left[k_{i-1}\left(\mathcal{G} \mathcal{R} \mathcal{A D} p_{h}\right)_{i-1} u_{i-1}+k_{i}\left(\mathcal{G} \mathcal{R} \mathcal{A D} p_{h}\right)_{i} u_{i}\right]=-\sum_{i=1}^{n} h_{i-1 / 2}\left(\mathcal{D} \mathcal{I} \mathcal{V}^{k} \mathbf{u}_{h}\right)_{i-1 / 2} p_{i-1 / 2}
$$

Now, the change of summation gives the typical finite difference formula for the gradient operator:

$$
\left(\mathcal{G R} \mathcal{A D} p_{h}\right)_{i}=-\frac{p_{i-1 / 2}-p_{i+1 / 2}}{\left(h_{i-1 / 2}+h_{i+1 / 2}\right) / 2},
$$

where $p_{1 / 2}=p_{N+1 / 2}=0$. Formula for the flux is

$$
k_{i} u_{i}=-k_{i}\left(\mathcal{G} \mathcal{R} \mathcal{A D} p_{h}\right)_{i}=k_{i} \frac{p_{i-1 / 2}-p_{i+1 / 2}}{\left(h_{i-1 / 2}+h_{i+1 / 2}\right) / 2}
$$

At this moment, we still have some freedom in selecting $k_{i}$. For instance, the conventional FV scheme is recovered for $k_{i}=k_{i}^{H}$. With the same assumption on the accuracy of $k_{i}$ as in the first scheme, we obtain the following truncation error on a non-smooth mesh:

$$
\left|(k u)\left(x_{i}\right)-k_{i} u_{i}\right| \leq C h\left(\left|p^{\prime \prime}\right|\right)+C h^{s}+O\left(h^{2}\right) .
$$

The truncation error on a smooth mesh is given by (3.3). Again, an accurate approximation of $k_{i}$ will lead to a better scheme. Note, that we can extend this scheme to multiple dimensions only in the case of simplicial meshes. Indeed, on a simplex, it is always possible to build a linear function that matches the given values $k_{i}$ at centers of simplex faces.

On a general polygon or a polyhedron such a construction is not possible. Instead, we consider a linear function that approximates $k$ in some sense. For example, we can use a linear reconstruction from the given cell-based values $\tilde{k}^{i-1 / 2}$. For our $1 \mathrm{D}$ example it means that we use values of the linear reconstruction at the cell end-points in the trapezoidal rule.

This gives two values $\tilde{k}_{i}^{i+1 / 2}$ and $\tilde{k}_{i}^{i-1 / 2}$ at each mesh node. The new discrete integration by parts formula reads:

$$
\sum_{i=1}^{n} \frac{h_{i-1 / 2}}{2}\left[\tilde{k}_{i-1}^{i-1 / 2}\left(\mathcal{G} \mathcal{R} \mathcal{A D} p_{h}\right)_{i-1} u_{i-1}+\tilde{k}_{i}^{i-1 / 2}\left(\mathcal{G} \mathcal{R} \mathcal{A D} p_{h}\right)_{i} u_{i}\right]=-\sum_{i=1}^{n} h_{i-1 / 2}\left(\mathcal{D} \mathcal{I} \mathcal{V}^{k} \mathbf{u}_{h}\right)_{i-1 / 2} p_{i-1 / 2}
$$


Explicit formula for the gradient is

$$
\left(\mathcal{G R} \mathcal{A D} p_{h}\right)_{i}=-\frac{k_{i}\left(h_{i-1 / 2}+h_{i+1 / 2}\right)}{h_{i-1 / 2} \tilde{k}_{i}^{i-1 / 2}+h_{i+1 / 2} \tilde{k}_{i}^{i+1 / 2}} \frac{p_{i-1 / 2}-p_{i+1 / 2}}{\left(h_{i-1 / 2}+h_{i+1 / 2}\right) / 2},
$$

where $p_{1 / 2}=p_{N+1 / 2}=0$. The truncation error analysis gives estimates (3.5) and (3.3) on non-smooth and smooth meshes, respectively.

Remark 3.1 Inserting formulas for the gradient in the divergence equation, we obtain a cell-centered scheme characterized by a tri-diagonal symmetric positive-definite matrix.

Remark 3.2 A few examples considered so far indicates that there exists many different inner products in the space of discrete gradients. The inner products based on the trapezoidal rule can be written as vector-matrix-vector products with diagonal matrices. In multiple dimensions, inner products represented by diagonal matrices are accurate approximations of $L^{2}$ dot products only on a handful of special meshes. On a general mesh, the approximation technology described in Section 4 leads to a non-diagonal symmetric positive definite matrix. Note, that inner products represented by non-diagonal $2 \times 2$ matrices can be introduced in $1 D$ too; however, the resulting schemes have no exceptional properties.

\subsubsection{Hybridization and scheme III}

In the case of discontinuous coefficient $k$, the gradients are also discontinuous. In such a case, the construction of an accurate inner product in the space of gradients becomes a nontrivial task, since a single number $u_{i}$ cannot represent accurately both left and right limits of function $u$. A hybridization technique solves this problem. It allows us to introduce two unknowns $u_{i}^{i-1 / 2}$ and $u_{i}^{i+1 / 2}$ (instead of $u_{i}$ ) and two values $k_{i}^{i-1 / 2}$ and $k_{i}^{i+1 / 2}$ (instead of $k_{i}$ ) at a discontinuity point and close the system of discrete equations by one additional equation:

$$
k_{i}^{i-1 / 2} u_{i}^{i-1 / 2}=k_{i}^{i+1 / 2} u_{i}^{i+1 / 2} .
$$

Moreover, for the efficient code implementation, we double gradient unknowns in all other mesh points and close the system of discrete equations with the same continuity equa-

tions. Note that it is common to have $k_{i}^{i-1 / 2}=k_{i}^{i+1 / 2}$ almost everywhere except for the discontinuity points.

Formula (3.6) is general enough to cover all schemes considered above. Using new notations, we write the primary divergence operator as

$$
\left(\widetilde{\mathcal{D I} \mathcal{V}}^{k} \mathbf{u}_{h}\right)_{i-1 / 2}=\frac{1}{h_{i-1 / 2}}\left(k_{i}^{i-1 / 2} u_{i}^{i-1 / 2}-k_{i-1}^{i-1 / 2} u_{i-1}^{i-1 / 2}\right), \quad 1 \leq i \leq n .
$$

Note that the fractional index labels the cell where the corresponding quantity resides. Due to the presence of linear constraints (3.6), we have to include them in the discrete integration by part formula:

$$
\begin{aligned}
\sum_{i=1}^{n} \frac{h_{i-1 / 2}}{2} & {\left[\tilde{k}_{i-1}^{i-1 / 2}\left(\widetilde{\mathcal{G R \mathcal { A } \mathcal { D }}} p_{h}\right)_{i-1}^{i-1 / 2} u_{i-1}^{i-1 / 2}+\tilde{k}_{i}^{i-1 / 2}\left(\widetilde{\mathcal{G \mathcal { R } \mathcal { A D }}} p_{h}\right)_{i}^{i-1 / 2} u_{i}^{i-1 / 2}\right]=} \\
& -\sum_{i=1}^{n} h_{i-1 / 2}\left(\widetilde{\mathcal{D I} \mathcal{V}}^{k} \mathbf{u}_{h}\right)_{i-1 / 2} p_{i-1 / 2}+\sum_{i=2}^{n} \lambda_{i}\left(k_{i}^{i-1 / 2} u_{i}^{i-1 / 2}-k_{i}^{i+1 / 2} u_{i}^{i+1 / 2}\right)
\end{aligned}
$$


where $\lambda_{i}$ are refereed to as the Lagrange multipliers. The change of summation gives

$$
\left.\left(\widetilde{\mathcal{G R} \mathcal{A} D} p_{h}\right)_{i}^{i-1 / 2}=-\frac{2 k_{i}^{i-1 / 2}}{\tilde{k}_{i}^{i-1 / 2}} \frac{p_{i-1 / 2}-\lambda_{i}}{h_{i-1 / 2}}, \quad \widetilde{(\mathcal{G \mathcal { A } \mathcal { A D }}} p_{h}\right)_{i}^{i+1 / 2}=-\frac{2 k_{i}^{i+1 / 2}}{\tilde{k}_{i}^{i+1 / 2}} \frac{\lambda_{i}-p_{i+1 / 2}}{h_{i+1 / 2}}
$$

The one-sided physical velocities are given by

$$
k_{i}^{i-1 / 2} u_{i}^{i-1 / 2}=-\frac{2\left(k_{i}^{i-1 / 2}\right)^{2}}{\tilde{k}_{i}^{i-1 / 2}} \frac{p_{i-1 / 2}-\lambda_{i}}{h_{i-1 / 2}}, \quad k_{i}^{i+1 / 2} u_{i}^{i+1 / 2}=-\frac{2\left(k_{i}^{i+1 / 2}\right)^{2}}{\tilde{k}_{i}^{i+1 / 2}} \frac{\lambda_{i}-p_{i+1 / 2}}{h_{i+1 / 2}} .
$$

The hybridization procedure is completed by substituting these formulas in the constraints (3.6) and the mass balance equations (3.7). Multiplying the mass balance equations by $h_{i-1 / 2}$, we obtain a system for pressure unknowns and Lagrange multipliers with a symmetric and positive definite matrix.

To compare this scheme with the previous schemes, we use constraint (3.6) to calculate the Lagrange multiplier as the function of two pressure unknowns, $\lambda_{i}=\lambda_{i}\left(p_{i-1 / 2}, p_{i+1 / 2}\right)$. Eliminating the Lagrange multiplier for the flux formulas, we obtain

$k_{i}^{i-1 / 2} u_{i}^{i-1 / 2}=k_{i}^{i+1 / 2} u_{i}^{i+1 / 2}=-\frac{\left(k_{i}^{i-1 / 2} k_{i}^{i+1 / 2}\right)^{2}\left(h_{i-1 / 2}+h_{i+1 / 2}\right)}{\left(k_{i}^{i-1 / 2}\right)^{2} \tilde{k}_{i}^{i+1 / 2} h_{i+1 / 2}+\left(k_{i}^{i+1 / 2}\right)^{2} \tilde{k}_{i}^{i-1 / 2} h_{i-1 / 2}} \frac{p_{i-1 / 2}-p_{i+1 / 2}}{\left(h_{i-1 / 2}+h_{i+1 / 2}\right) / 2}$.

When $k_{i}^{i-1 / 2}=k_{i}^{i+1 / 2}=k_{i}$ we recover scheme II with linear approximation of the diffusion coefficient inside mesh cells. If in addition $\tilde{k}_{i-1}^{i-1 / 2}=\tilde{k}_{i}^{i-1 / 2}=\tilde{k}^{i-1 / 2}$, we recover scheme I. In the next section we extend the hybridization procedure to multiple dimensions.

\subsection{Mimetic schemes in 2D and 3D}

\subsubsection{Discrete spaces and interpolation operators}

The discrete pressure space $\mathcal{P}_{h}$ consists of one degree of freedom per cell corresponding to the pressure value at the center of mass. The dimension of $\mathcal{P}_{h}$ equals to the number of mesh cells. For vector $p_{h} \in \mathcal{P}_{h}$, we shall denote by $p_{c}$ its value on cell $c$. For a given integrable scalar function $p$, we denote by $p^{I} \in \mathcal{P}_{h}$ the vector of degrees of freedom such that

$$
p^{I}=\left\{p_{c}^{I}\right\}_{c \in \Omega_{h}}, \quad p_{c}^{I}=\frac{1}{|c|} \int_{c} p d x .
$$

The discrete space $\mathcal{F}_{h}$ consists of one degree of freedom per boundary face and two degrees of freedom per interior face. For vector $\mathbf{u}_{h} \in \mathcal{F}_{h}$, we denote by $\mathbf{u}_{c}$ its restriction to cell $c$, and by $u_{f}^{c}$ its component associated with face $f$ of cell $c$ (analog of the onedimensional quantity $\left.u_{i}^{i-1 / 2}\right)$. Hereafter, we consider a subspace of $\mathcal{F}_{h}$ whose members satisfy the flux continuity constraint

$$
k_{f}^{c_{1}} u_{f}^{c_{1}}=k_{f}^{c_{2}} u_{f}^{c_{2}}
$$

on each interior face $f$ shared by cells $c_{1}$ and $c_{2}$. Like in one dimension, we may set $k_{f}^{c_{1}}=k_{f}^{c_{2}}$ on mesh faces where the diffusion coefficient is continuous; although the sufficient condition for having an accurate scheme is weaker, $k_{f}^{c_{1}}-k_{f}^{c_{2}}=O\left(h^{2}\right)$. 
For a given sufficiently smooth (inside mesh cell) vector function $\mathbf{u}$, we define $\mathbf{u}^{I} \in \mathcal{F}_{h}$ as a vector of degrees of freedom such that

$$
\mathbf{u}^{I}=\left\{\mathbf{u}_{c}^{I}\right\}_{c \in \Omega_{h}}, \quad \mathbf{u}_{c}^{I}=\left\{\left(u_{f}^{c}\right)^{I}\right\}_{f \in \partial c}, \quad\left(u_{f}^{c}\right)^{I}=\frac{1}{|f|} \int_{f} \mathbf{u}_{\left.\right|_{c}} \cdot \mathbf{n}_{f} d x
$$

where $\mathbf{n}_{f}$ is a unit face normal that is fixed once and for all, and $\mathbf{u}_{\mid c} \cdot \mathbf{n}_{f}$ is the one-sided limit from inside cell $c$.

\subsubsection{Primary mimetic operator}

The primary divergence operator is defined locally on each mesh cell using a straightforward discretization of the divergence theorem:

$$
\left.\left(\mathcal{D} \mathcal{I} \mathcal{V}^{k} \mathbf{u}_{h}\right)\right|_{c} \equiv \mathcal{D} \mathcal{I} \mathcal{V}_{c}^{k} \mathbf{u}_{c}=\frac{1}{|c|} \sum_{f \in \partial c} \sigma_{c, f}|f| k_{f}^{c} u_{f}^{c}
$$

where $\sigma_{c, f}$ is either 1 or -1 depending on the mutual orientation of the fixed normal $\mathbf{n}_{f}$ and the exterior normal to $\partial c$. Extrapolating from the one dimensional results, we assume that $k_{f}^{c}$ is an accurate (one-sided) approximation of the diffusion coefficient on face $f$. For example, $k_{f}^{c}$ could be defined by some averaging of cell-centered values of the diffusion coefficient.

Since $\mathbf{u}_{h}$ is an algebraic vector, it is convenient to think about the discrete divergence operator $\mathcal{D} \mathcal{I} \mathcal{V}^{k}: \mathcal{F}_{h} \rightarrow \mathcal{P}_{h}$ as a matrix acting between two spaces. Note that this matrix has the full rank when $k_{f}^{c}>0$.

Let us introduce a cell-based diagonal matrix $\mathcal{K}_{c}$ formed by coefficients $k_{f}^{c}, f \in \partial c$. Then, the primary divergence operator can be written using the conventional divergence operator, the property can be used in the efficient computer implementation of new schemes:

$$
\mathcal{D} \mathcal{I} \mathcal{V}_{c}^{k} \mathbf{u}_{c}=\left(\mathcal{D} \mathcal{I} \mathcal{V}_{c} \mathcal{K}_{c}\right) \mathbf{u}_{c}, \quad \mathcal{D} \mathcal{I} \mathcal{V}_{c} \mathbf{u}_{c}=\frac{1}{|c|} \sum_{f \in \partial c} \sigma_{c, f}|f| u_{f}^{c}
$$

\subsubsection{Derived mimetic operator: continuous diffusion coefficient}

In this subsection, we extend schemes I and II to multiple dimensions. Hence, we assume that $k_{f}^{c_{1}}=k_{f}^{c_{2}}=k_{f}$ on all interior mesh faces. The derived mimetic operator should be a consistent approximation of operator $\nabla$ in the following sense. For $k \in L^{\infty}(\Omega)$, $k \mathbf{u} \in H_{\text {div }}(\Omega)$ and $p \in H_{0}^{1}(\Omega)$, we consider the continuum integration by parts formula

$$
\int_{\Omega} k(\nabla p) \cdot \mathbf{u} d x=-\int_{\Omega}(\operatorname{div} k \mathbf{u}) p d x .
$$

The formula implies that operator div $k$ is negatively adjoint to operator $\nabla$ with respect to the weighted $L^{2}$ products. To mimic this property in a discrete setting, we replace the integrals by their accurate approximations. For the first integral, we require

$$
\left[\mathbf{v}^{I}, \mathbf{u}^{I}\right]_{\mathcal{F}_{h}}=\int_{\Omega} k \mathbf{v} \cdot \mathbf{u} d x+O(h)
$$


where vectors $\mathbf{v}^{I}, \mathbf{u}^{I} \in \mathcal{F}_{h}$ are discrete representations of vector functions $\mathbf{v}, \mathbf{u}$, respectively. Similarly, for scalar functions we require that

$$
\left[q^{I}, p^{I}\right]_{\mathcal{P}_{h}}=\int_{\Omega} q p d x+O(h) .
$$

We assume that the inner products are well defined, i.e. they can be represented by symmetric positive definite matrices $\mathbb{M}_{\mathcal{F}}$ and $\mathbb{M}_{\mathcal{P}}$ (see Section 3.3 for the derivation of these matrices):

$$
\left[\mathbf{v}_{h}, \mathbf{u}_{h}\right]_{\mathcal{F}_{h}}=\left(\mathbf{v}_{h}\right)^{T} \mathbb{M}_{\mathcal{F}} \mathbf{u}_{h}, \quad\left[q_{h}, p_{h}\right]_{\mathcal{P}_{h}}=\left(q_{h}\right)^{T} \mathbb{M}_{\mathcal{P}} p_{h}
$$

With these notations, the derived mimetic operators $\mathcal{G R} \mathcal{A D}$ is defined as the operator that satisfies the discrete integration by parts formula:

$$
\left[\mathcal{G} \mathcal{R} \mathcal{A D} p_{h}, \mathbf{u}_{h}\right]_{\mathcal{F}_{h}}=-\left[\mathcal{D} \mathcal{I} \mathcal{V}^{k} \mathbf{u}_{h}, p_{h}\right]_{\mathcal{P}_{h}} \quad \forall \mathbf{u}_{h} \in \mathcal{F}_{h}, p_{h} \in \mathcal{P}_{h}
$$

Inserting definitions (3.9) into this formula and using arbitrariness of vectors $\mathbf{u}_{h}$ and $p_{h}$, we obtain the explicit matrix representation of the derived gradient operator:

$$
\mathcal{G} \mathcal{R} \mathcal{A D}=-\mathbb{M}_{\mathcal{F}}^{-1}\left(\mathcal{D} \mathcal{I} \mathcal{V}^{k}\right)^{T} \mathbb{M}_{\mathcal{P}}
$$

Note that matrix $\mathbb{M}_{\mathcal{F}}$ is in general irreducible; hence, in contrast to $1 \mathrm{D}$ schemes, the derived operator on an unstructured 2D or 3D mesh has a non-local stencil. This property is shared by all known accurate discretizations of mixed diffusion formulations.

Combining the two equations in (3.1) (with $a=0$ ) and multiplying both sides by matrix $\mathbb{M}_{\mathcal{P}}$ we obtain a system of discrete equations with a symmetric positive definite matrix:

$$
\mathbb{M}_{\mathcal{P}} \mathcal{D} \mathcal{I} \mathcal{V}^{k} \mathbb{M}_{\mathcal{F}}^{-1}\left(\mathcal{D} \mathcal{I} \mathcal{V}^{k}\right)^{T} \mathbb{M}_{\mathcal{P}} p_{h}=\mathbb{M}_{\mathcal{P}} b^{I}
$$

The symmetry property is due to the discrete integration by part property, while the positive definiteness property is due to the full rank of the discrete divergence matrix.

Thus, the new mimetic scheme preserves critical properties of the continuum problem: symmetry and positive-definiteness. In contrast to the classical mimetic scheme, now we have freedom of using staggered discretization of the diffusion coefficient in mesh cells and on mesh faces.

Remark 3.3 The mimetic primary-derived technology can be extended to the case of tensorial diffusion coefficients in the form $k \mathbb{K}$ where $\mathbb{K}$ is a tensorial factor. The primary mimetic operator $\mathcal{D} \mathcal{I} \mathcal{V}^{k}$ remains the same. The derived mimetic operator $\mathcal{G} \mathcal{R} \mathcal{A D}$ approximates the continuum operator $\mathbb{K} \nabla$. Its derivation is based on the following integration by parts formula:

$$
\int_{\Omega} \mathbb{K}^{-1} k(\mathbb{K} \nabla p) \cdot \mathbf{u} d x=-\int_{\Omega}(\operatorname{div} k \mathbf{u}) p d x \quad \forall \mathbf{u} \in H_{\text {div }}(\Omega), p \in H_{0}^{1}(\Omega) .
$$

To approximate the first integral, it is sufficient to replace $\mathbb{K}$ by a piece-wise constant tensor. 


\subsubsection{Derived mimetic operator: discontinuous diffusion coefficient}

In this subsection, we extend scheme III to multiple dimensions. Although the approach used in Section 3.1.3 can be extended to multiple dimensions, we choose a different strategy which provides more insight on the physical meaning of the Lagrange multipliers. Consider the continuum integration by parts formula in cell $c$ :

$$
\int_{c} k(\nabla p) \cdot \mathbf{u} d x=-\int_{c}(\operatorname{div} k \mathbf{u}) p d x+\int_{\partial c}(k \mathbf{u}) \cdot \mathbf{n} p d x
$$

It implies that a cell-based discrete gradient must use additional pressure unknowns on mesh faces. We define them as $\lambda_{f}$ :

$$
\lambda_{f} \approx \frac{1}{|f|} \int_{f} p d x
$$

Let $\lambda_{c}$ be a cell-based vector of such unknowns ordered similarly to the unknowns in $\mathbf{u}_{c}$. Using the above formulas, we postulate the following cell-based discrete integration by parts formula:

$$
\left[\mathcal{G} \mathcal{R} \mathcal{A D}_{c}\left(\begin{array}{c}
p_{c} \\
\lambda_{c}
\end{array}\right), \mathbf{u}_{c}\right]_{c, \mathcal{F}_{h}}=-\left[\mathcal{D} \mathcal{I} \mathcal{V}_{c}^{k} \mathbf{u}_{c}, p_{c}\right]_{c, \mathcal{P}_{h}}+\sum_{f \in \partial c} \sigma_{c, f}|f| k_{f}^{c} u_{f}^{c} \lambda_{f} \quad \forall \mathbf{u}_{c}, p_{c}, \lambda_{c}
$$

Due to constraints (3.8), summation of formulas (3.13) over mesh cells leads to cancellation of $\lambda$-terms in the right hand side on all internal faces. On boundary faces, these terms are zeroed-out due to homogeneous boundary conditions. Hence, the global gradient coincides with the gradient operator introduced in the previous section provided that the same algorithm is used for constructing inner products.

Introduction of additional pressure unknowns allows us to write an explicit formula for the cell-based gradient using a cell-based mass matrix $\mathbb{M}_{c, \mathcal{F}}$ that represents the inner product $[\cdot, \cdot]_{c, \mathcal{F}_{h}}$ :

$$
\mathcal{G} \mathcal{R} \mathcal{A D}_{c}\left(\begin{array}{c}
p_{c} \\
\lambda_{c}
\end{array}\right)=-\mathbb{M}_{c, \mathcal{F}}^{-1}\left(\begin{array}{c}
\sigma_{c, f_{1}} k_{f_{1}}^{c}\left|f_{1}\right|\left(p_{c}-\lambda_{f_{1}}\right) \\
\vdots \\
\sigma_{c, f_{n}} k_{f_{n}}^{c}\left|f_{n}\right|\left(p_{c}-\lambda_{f_{n}}\right)
\end{array}\right)
$$

where $n$ is the number of cell faces. Formally, for a general mesh we should use the symbol $n_{c}$; however, subsequent discussion is focused on a single cell. The formula for physical fluxes becomes:

$$
\mathcal{K}_{c}\left(\begin{array}{c}
u_{f_{1}}^{c} \\
\vdots \\
u_{f_{n}}^{c}
\end{array}\right)=-\mathcal{K}_{c} \mathbb{M}_{c, \mathcal{F}}^{-1} \mathcal{K}_{c}\left(\begin{array}{c}
\sigma_{c, f_{1}}\left|f_{1}\right|\left(p_{c}-\lambda_{f_{1}}\right) \\
\vdots \\
\sigma_{c, f_{n}}\left|f_{n}\right|\left(p_{c}-\lambda_{f_{n}}\right)
\end{array}\right)
$$

Now, the reason for introducing local gradients becomes clear. Inserting formulas for the cell-based gradient into constraints (3.8) and the mass balance equations and multiplying 
the latter equations by matrix $\mathbb{M}_{\mathcal{P}}$, we obtain a system of algebraic equations with respect to cell-based and face-based pressure unknowns only:

$$
\mathbb{A}\left(\begin{array}{c}
p_{h} \\
\lambda_{h}
\end{array}\right)=\left(\begin{array}{c}
\mathbb{M}_{\mathcal{P}} b^{I} \\
0
\end{array}\right)
$$

subject to the homogeneous boundary conditions, $\lambda_{f}=0$ for $f \in \partial \Omega$. It could be shown that stiffness matrix $\mathbb{A}$ can be assembled from cell-based stiffness matrices:

$$
\left(\begin{array}{c}
q_{h} \\
\mu_{h}
\end{array}\right)^{T} \mathbb{A}\left(\begin{array}{c}
p_{h} \\
\lambda_{h}
\end{array}\right)=\sum_{c \in \Omega_{h}}\left(\begin{array}{c}
q_{c} \\
\mu_{c}
\end{array}\right)^{T} \mathbb{A}_{c}\left(\begin{array}{c}
p_{c} \\
\lambda_{c}
\end{array}\right) .
$$

The block structure of each matrix $\mathbb{A}_{c}$ is standard for all mimetic schemes and differs by the definition of matrix $\mathbb{W}_{c}$, which is convenient for a computer implementation of new schemes:

$$
\mathbb{A}_{c}=\left(\begin{array}{cc}
\mathbf{e}^{T} \mathbb{W}_{c} \mathbf{e} & -\mathbf{e}^{T} \mathbb{W}_{c} \\
-\mathbb{W}_{c} \mathbf{e} & \mathbb{W}_{c}
\end{array}\right)
$$

where $\mathbf{e}=\left(\left|f_{1}\right|, \ldots,\left|f_{n}\right|\right)^{T}$ and $\mathbb{W}_{c}=\mathcal{K}_{c} \mathbb{M}_{c, \mathcal{F}}^{-1} \mathcal{K}_{c}$. The difference between schemes I and II is in definition of matrix $\mathbb{M}_{c, \mathcal{F}}$. The difference between schemes II and III is in the definition of matrix $\mathcal{K}_{c}$.

Assembling of local matrices leads to a global matrix with a symmetric positive semidefinite matrix. Enforcement of essential boundary conditions, makes this matrix positive definite. That is why the hybridization procedure is the most efficient way for implementing mimetic schemes. After solving for pressure unknowns, the fluxes are calculated using formula (3.15). In all steps of the solution algorithm, we need only the inverse of the elemental mass matrix $\mathbb{M}_{c, \mathcal{F}}$. An efficient algorithm for the direct calculation of the inverse matrix is described later.

\subsubsection{Boundary conditions}

Non-homogeneous Dirichlet boundary conditions could be embedded into the definition of space $\mathcal{P}_{h}$. Although, here we advocate the hybrid mimetic scheme that uses Lagrange multipliers. The Dirichlet boundary conditions are imposed on the Lagrange multipliers.

Neumann boundary conditions are essential conditions for fluxes $k_{f}^{c} u_{f}^{c}$ and can be easily added as constraint conditions on boundary faces, similarly to formula (3.8). In a hybrid approach, this leads to a more homogeneous computer implementation of the mimetic schemes.

\subsection{Accurate inner products in spaces $\mathcal{P}_{h}$ and $\mathcal{F}_{h}$}

The inner product in space $\mathcal{P}_{h}$ is build by assembling cell-based inner products. This leads to a significant simplification of construction, since we have only one degree of freedom in cell $c$. The explicit formula of the inner product is

$$
\left[q^{I}, p^{I}\right]_{\mathcal{P}_{h}}=\sum_{c \in \Omega_{h}}\left[q_{c}^{I}, p_{c}^{I}\right]_{c, \mathcal{P}_{h}}, \quad\left[q_{c}^{I}, p_{c}^{I}\right]_{c, \mathcal{P}_{h}}=|c| q_{c}^{I} p_{c}^{I}=\int_{c} q p d x+O\left(h_{c}|c|\right)
$$


This implies that the mass matrix $\mathbb{M}_{\mathcal{P}}$ is diagonal with values $|c|$ on the diagonal.

Similarly, the inner product in space $\mathcal{F}_{h}$ is build by assembling accurate cell-based inner-products which mimics additivity of integration:

$$
\left[\mathbf{v}^{I}, \mathbf{u}^{I}\right]_{\mathcal{F}_{h}}=\sum_{c \in \Omega_{h}}\left[\mathbf{v}_{c}^{I}, \mathbf{u}_{c}^{I}\right]_{c, \mathcal{F}_{h}}, \quad\left[\mathbf{v}_{c}^{I}, \mathbf{u}_{c}^{I}\right]_{c, \mathcal{F}_{h}}=\int_{c} k \mathbf{v} \cdot \mathbf{u} d x+O\left(h_{c}|c|\right) .
$$

To build an accurate approximation of the cell-based inner product, we can make a few approximation steps. First, we replace vector function $\mathbf{v}$ by its best constant approximation $\mathbf{v}_{0}$ (e.g., the $L^{2}$ projection) which leads to an admissible error of order $h_{c}$. Second, we approximate function $k$ by a linear function $\tilde{k}_{1}$ (a constant approximation will work too). We use symbol tilde for analogy with the one-dimensional schemes. Third, we approximate function $\mathbf{u}$ by a function (still denoted by $\mathbf{u}$ for simplicity of exposition) that has two special properties: (a) $\mathbf{u} \cdot \mathbf{n}$ is constant on each faces $f$ of cell $c$, and (b) $\operatorname{div}(\mathbf{u})$ is constant in $c$. The space of such functions is denoted by $\mathcal{S}(c)$. It is sufficiently rich and contains the space of constant vector functions, so that we make an admissible approximation error. The interpolants $\mathbf{u}^{I}$ cover the whole space of cell-based discrete gradients, which is important for the convergence analysis. Thus, we need to find a symmetric positive definite matrix $\mathbb{M}_{c, \mathcal{F}_{h}}$ such that

$$
\left(\mathbf{v}_{0}^{I}\right)^{T} \mathbb{M}_{c, \mathcal{F}} \mathbf{u}_{c}^{I}=\int_{c} \tilde{k}_{1} \mathbf{v}_{0} \cdot \mathbf{u} d x+O\left(h_{c}|c|\right)
$$

for any constant $\mathbf{v}_{0}$, linear $\tilde{k}_{1}$ and $\mathbf{u} \in \mathcal{S}(c)$. We can write $\mathbf{v}^{0}=\nabla p_{1}$ where $p_{1}$ is a linear function such that $p_{1}\left(\mathbf{x}_{c}\right)=0$. Inserting it in the right-hand side of (3.17) and integrating by parts, we obtain

$$
\int_{c} \tilde{k}_{1} \nabla p_{1} \cdot \mathbf{u} d x=-\int_{c}\left(\operatorname{div} \tilde{k}_{1} \mathbf{u}\right) p_{1} d x+\int_{\partial c} \tilde{k}_{1}(\mathbf{u} \cdot \mathbf{n}) p_{1} d x
$$

The volume integral in the right-hand side is of high-order with respect to the surface integral. Indeed, using $\operatorname{div}\left(\tilde{k}_{1} \mathbf{u}\right)=\tilde{k}_{1} \operatorname{div}(\mathbf{u})+\nabla \tilde{k}_{1} \cdot \mathbf{u}$ and that $\operatorname{div}(\mathbf{u})$ is constant, we have

$$
\int_{c} \operatorname{div}\left(\tilde{k}_{1} \mathbf{u}\right) p_{1} d x=\int_{c} \nabla \tilde{k}_{1} \cdot\left(\mathbf{u}-\mathbf{u}_{0}\right) p_{1} d x=O\left(h_{c}|c|\right),
$$

where $\mathbf{u}_{0}$ is the best constant approximation of $\mathbf{u}$. By our assumptions, $\mathbf{u} \cdot \mathbf{n}_{f}$ is constant on face $f$ and can be pulled out of the face integrals. Finally, we can define the inner product matrix from

$$
\left(\left(\nabla p_{1}\right)_{c}^{I}\right)^{T} \mathbb{M}_{c, \mathcal{F}} \mathbf{u}_{c}^{I}=\sum_{f \in \partial c} u_{f}^{c} \sigma_{c, f} \int_{f} \tilde{k}_{1} p_{1} d x \quad \forall p_{1}, \forall \mathbf{u} \in \mathcal{S}(c) .
$$

Now, we use the linearity of the space of linear functions $p_{1}$ to get an alternative representation of equation (3.19). Let us introduce the cell-based vector $\mathbf{r}_{c}=\mathbf{r}_{c}\left(p_{1}\right)$ with the following entries:

$$
\mathbf{r}_{c}=\left\{r_{f}^{c}\right\}_{f \in \partial c}, \quad r_{f}^{c}=\sigma_{c, f} \int_{f} \tilde{k}_{1} p_{1} d x
$$


Since $\tilde{k}_{1}$ and $p_{1}$ are linear functions, we can use the Simpson quadrature rule in 2D. In 3D, we can reduce this integration to the integration over $\partial f$ using the divergence theorem. We define matrix $\mathbb{M}_{c, \mathcal{F}}$ as a solution of the system of matrix equations:

$$
\mathbb{M}_{c, \mathcal{F}}\left(\nabla p_{1}\right)_{c}^{I}=\mathbf{r}_{c}\left(p_{1}\right) \quad \forall p_{1} .
$$

Due to the linearity of these equations, it is sufficient to consider only three linearly independent functions in 3D: $p_{1, x}=x-x_{c}, p_{1, y}=y-y_{c}$, and $p_{1, z}=z-z_{c}$. Let

$$
\mathbb{N}_{c}=\left[\left(\nabla p_{1, x}\right)_{c}^{I}\left(\nabla p_{1, y}\right)_{c}^{I}\left(\nabla p_{1, z}\right)_{c}^{I}\right], \quad \mathbb{R}_{c}=\left[\mathbf{r}_{c}\left(p_{1, x}\right) \mathbf{r}_{c}\left(p_{1, y}\right) \mathbf{r}_{c}\left(p_{1, z}\right)\right]
$$

be two rectangular $n \times 3$ matrices. The matrix equation (3.20) is equivalent to

$$
\mathbb{M}_{c, \mathcal{F}} \mathbb{N}_{c}=\mathbb{R}_{c}
$$

A simple linear algebra allows us to write the explicit formula (which could be verified by a direct calculation) for matrix $\mathbb{M}_{c, \mathcal{F}}$ :

$$
\mathbb{M}_{c, \mathcal{F}}=\mathbb{R}_{c}\left(\mathbb{R}_{c}^{T} \mathbb{N}_{c}\right)^{-1} \mathbb{R}_{c}^{T}+\gamma_{c} \mathbb{P}_{c}, \quad \mathbb{P}_{c}=\mathbb{I}-\mathbb{N}_{c}\left(\mathbb{N}_{c}^{T} \mathbb{N}_{c}\right)^{-1} \mathbb{N}_{c}^{T}
$$

with a positive factor $\gamma_{c}$ in front of the projection matrix $\mathbb{P}_{c}$. A recommended choice for $\gamma_{c}$ is the mean trace of the first term. A family of mimetic schemes is obtained if we replace $\gamma_{c}$ by an arbitrarily symmetric positive definite matrix $\mathbb{G}_{c}$ :

$$
\mathbb{M}_{c, \mathcal{F}}=\mathbb{R}_{c}\left(\mathbb{R}_{c}^{T} \mathbb{N}_{c}\right)^{-1} \mathbb{R}_{c}^{T}+\mathbb{P}_{c} \mathbb{G}_{c} \mathbb{P}_{c} .
$$

Stability of the resulting mimetic scheme depends on two factors: (a) the spectral bounds of matrix $\mathbb{G}_{c}$ that should be close to the value of $\gamma_{c}$, and (b) the properties of matrix-matrix product $\mathbb{R}_{c}^{T} \mathbb{N}_{c}$. Let $\tilde{k}_{1}$ be a linear function over cell $c$. The product of the first columns of matrices $\mathbb{N}_{c}$ and $\mathbb{R}_{c}$ is

$$
\begin{aligned}
\left.\left(\nabla p_{1, x}\right)_{c}^{I}\right)^{T} \mathbf{r}_{c}\left(p_{1, x}\right) & \left.=\left(\nabla p_{1, x}\right)_{c}^{I}\right)^{T} \mathbb{M}_{c, \mathcal{F}}\left(\nabla p_{1, x}\right)_{c}^{I}=\int_{\partial c}\left(\nabla p_{1, x} \cdot \mathbf{n}\right) \tilde{k}_{1} p_{1, x} d x \\
& =\int_{c} \tilde{k}_{1} \nabla p_{1, x} \cdot \nabla p_{1, x} d x=\int_{c} \tilde{k}_{1} d x=\tilde{k}_{1}\left(\mathbf{x}_{c}\right)|c|
\end{aligned}
$$

A similar argument works for the dot products of other columns. Thus, the matrix-matrix product $\mathbb{N}_{c}^{T} \mathbb{R}_{c}$ is well conditioned and does not depend on the gradient of $\tilde{k}_{1}$ :

$$
\mathbb{N}_{c}^{T} \mathbb{R}_{c}=\mathbb{I} \int_{c} \tilde{k}_{1} d x=\tilde{k}_{1}\left(\mathbf{x}_{c}\right)|c| \mathbb{I} .
$$

Remark 3.4 Consider the following matrix equation

$$
\mathbb{W}_{c, \mathcal{F}} \mathbb{R}_{c}=\mathbb{N}_{c}
$$

The solution of this equation is the inverse of one of the matrices $\mathbb{M}_{c, \mathcal{F}}$. Only this matrix is needed in the hybridization procedure. A general solution of this equation is given by

$$
\mathbb{W}_{c, \mathcal{F}}=\mathbb{N}_{c}\left(\mathbb{R}_{c}^{T} \mathbb{N}_{c}\right)^{-1} \mathbb{N}_{c}^{T}+\widetilde{\mathbb{P}}_{c} \widetilde{\mathbb{G}}_{c} \widetilde{\mathbb{P}}_{c},
$$

where $\widetilde{\mathbb{P}}_{c}$ is the projection matrix on the null space of matrix $\mathbb{R}_{c}^{T}$.

Remark 3.5 Note that the first term in $\mathbb{W}_{c, \mathcal{F}}$ dependents on $\tilde{k}_{1}\left(\mathbf{x}_{c}\right)$ and does not depend on the gradient of $\tilde{k}_{1}$. For any choice of the gradient, we obtain a family of convergent schemes. This flexibility enriches a set of the existing mimetic schemes and can be used in various optimization procedures [10]. 


\section{$4 \quad$ Numerical experiments}

Let us introduce the following relative errors for pressure and flux:

$$
\operatorname{err}(p)=\frac{\left.\left\|p^{I}-p_{h}\right\|\right|_{\mathcal{P}_{h}}}{\|\| p^{I} \|_{\mathcal{P}_{h}}}, \quad \operatorname{err}(\mathbf{u})=\frac{\left\|\mathcal{K}\left(\mathbf{u}^{I}-\mathbf{u}_{h}\right)\right\|_{2}}{\left\|\mathcal{K} \mathbf{u}^{I}\right\|_{2}}
$$

where \|\|$\cdot \|\left.\right|_{\mathcal{P}_{h}}$ is the norm induced by the inner product on $\mathcal{P}_{h},\|\cdot\|_{2}$ is the Euclidean norm and

$$
\left\|\mathcal{K} \mathbf{u}^{I}\right\|_{2}^{2}=\sum_{c \in \Omega_{h}}\left\|\mathcal{K}_{c} \mathbf{u}_{c}^{I}\right\|_{2}^{2}
$$

For the quasi-uniform meshes considered in the numerical experiments, the Euclidean norm leads to the same conclusions as any reasonable mesh-dependent $L^{2}$ norm.

Let us summarize the schemes that will be compared in this section. Scheme I uses one value, $k_{f}$, per mesh face (in the definition of the discrete divergence operator) and piecewise constant approximation, $k_{0}$, of the diffusion coefficient on mesh $\Omega_{h}$. Scheme II is similar to scheme I but uses the piecewise linear approximation, $k_{1}$, of the diffusion coefficient. In both schemes, coefficient $k_{f}$ is calculated by averaging traces of either $k_{0}$ or $k_{1}$ on mesh face $f$. Scheme III is similar to scheme I but uses two values $k_{f}^{c_{1}}$ and $k_{f}^{c_{2}}$ on faces where the diffusion coefficient is discontinuous. These coefficients are set to values of $k_{0}$ at the mid-point of face $f$.

\subsection{A sequence of $2 \mathrm{D}$ meshes and $3 \mathrm{D}$ meshes}

The numerical analysis is performed on a sequence of smooth and randomly perturbed quadrilateral meshes, and a sequence of polygonal meshes, see Fig. 3. The smooth mesh is obtained with the following mapping of vertex coordinates in a square mesh:

$$
\mathbf{x}:=\mathbf{x}+0.1 \sin (2 \pi x) \sin (2 \pi y) \mathbf{e}
$$

where $e=(1,1)^{T}$. The randomly perturbed mesh is obtained by a random shift of vertices in a square mesh. The new position of the mesh vertex can be anywhere inside a box of size $0.8 h$ centered at the original vertex. The polygonal mesh is built in two steps. First, we generate matching Delaunay meshes in the left and right parts of $\Omega$. Second, we build a constraint Voronoi tessellation in each subdomain.

The sequence of smooth hexahedral meshes is built similar to the sequence of smooth quadrilateral meshes. More precisely, we modify formula (4.1) by adding factor $\sin (2 \pi z)$ to the second term and use the result to build a hexahedral mesh.

\subsection{Exactness on linear solutions}

For a discontinuous piecewise-constant diffusion coefficient, only scheme III is exact for piecewise linear solutions. Indeed, it could be shown that all steps in the scheme derivation are linearity preserving. The other schemes are first-order accurate for pressure and velocity in the discrete $L^{2}$ norms. 

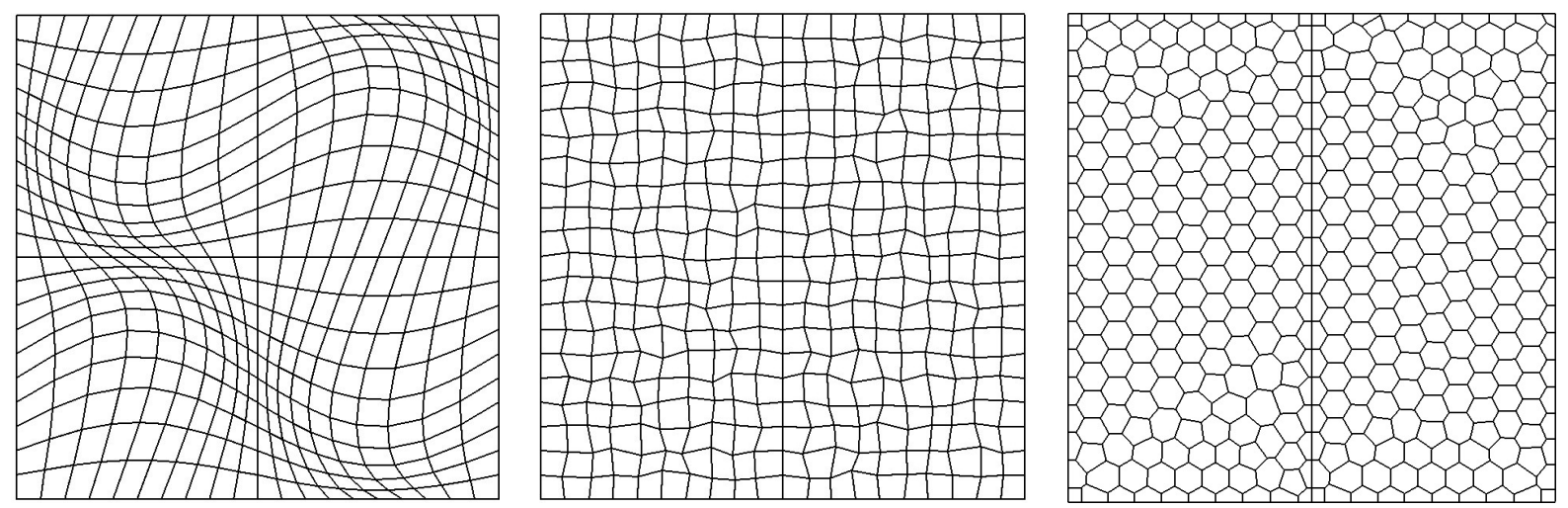

Figure 3: The first meshes in the three sequences of meshes.

Table 1: Continuous $k$ and sequence of smooth meshes (Fig. 3, left)

\begin{tabular}{c|cc|cc|cc}
$1 / h$ & \multicolumn{2}{|c|}{ Scheme I } & \multicolumn{2}{c|}{ Scheme II } & \multicolumn{2}{c}{ Standard MFD } \\
\hline & $\operatorname{err}(p)$ & $\operatorname{err}(\mathbf{u})$ & $\operatorname{err}(p)$ & $\operatorname{err}(\mathbf{u})$ & $\operatorname{err}(p)$ & $\operatorname{err}(\mathbf{u})$ \\
\hline 20 & $1.375 \mathrm{e}-3$ & $5.063 \mathrm{e}-3$ & $3.103 \mathrm{e}-3$ & $6.789 \mathrm{e}-3$ & $2.047 \mathrm{e}-3$ & $5.058 \mathrm{e}-3$ \\
40 & $3.480 \mathrm{e}-4$ & $1.358 \mathrm{e}-3$ & $8.601 \mathrm{e}-4$ & $1.827 \mathrm{e}-3$ & $5.205 \mathrm{e}-4$ & $1.312 \mathrm{e}-3$ \\
80 & $8.659 \mathrm{e}-5$ & $3.836 \mathrm{e}-4$ & $2.037 \mathrm{e}-4$ & $4.915 \mathrm{e}-4$ & $1.307 \mathrm{e}-4$ & $3.322 \mathrm{e}-4$ \\
160 & $2.154 \mathrm{e}-5$ & $1.143 \mathrm{e}-4$ & $5.103 \mathrm{e}-5$ & $1.379 \mathrm{e}-4$ & $3.273 \mathrm{e}-5$ & $8.369 \mathrm{e}-5$ \\
320 & $5.366 \mathrm{e}-6$ & $3.606 \mathrm{e}-5$ & $1.276 \mathrm{e}-5$ & $4.095 \mathrm{e}-5$ & $8.184 \mathrm{e}-6$ & $2.107 \mathrm{e}-5$ \\
\hline rate & 2.00 & 1.78 & 1.99 & 1.85 & 1.99 & 1.98
\end{tabular}

\subsection{Numerical comparison of MFD schemes in 2D}

We consider the following analytic solution

$p(x, y)=\left\{\begin{array}{ll}a_{1} x^{2}+y^{2}, & x<0.5, \\ a_{2} x^{2}+y^{2}+\frac{1}{4}\left(a_{1}-a_{2}\right), & x>0.5,\end{array} \quad k(x, y)= \begin{cases}b_{1}(1+x \sin (y)), & x<0.5, \\ b_{2}\left(1+2 x^{2} \sin (y)\right), & x>0.5,\end{cases}\right.$

where $a_{i} b_{i}=1$. We consider two problems with continuous and discontinuous function $k$. For the first problem, we set $b_{1}=b_{2}=1$. For the second problem, we set $b_{1}=1$ and $b_{2}=20$, so that the analytic solution has continuous normal velocity across interface $x=0.5$ and discontinuous tangential velocity.

Tables $1-3$ show the optimal first-order convergence for the flux and second-order convergence (superconvergence) for the pressure in the discrete norms. Note that a more accurate representation of $k$ inside mesh cells does not give us obvious benefits. The new schemes are competitive with the standard MFD scheme [13]. Since all schemes preserve a linear solution, the only difference we can expect is the magnitude of the error and not its decrease rate.

In Tables 4-6, we replaced scheme II with scheme III, since the first two schemes exhibit similar convergence properties. Scheme I shows degradation in the convergence rate due to 
Table 2: Continuous $k$ and sequence of randomly perturbed meshes (Fig. 3, center)

\begin{tabular}{c|cc|cc|cc}
$1 / h$ & \multicolumn{2}{|c|}{ Scheme I } & \multicolumn{2}{c|}{ Scheme II } & \multicolumn{2}{c}{ Standard MFD } \\
\hline & $\operatorname{err}(p)$ & $\operatorname{err}(\mathbf{u})$ & $\operatorname{err}(p)$ & $\operatorname{err}(\mathbf{u})$ & $\operatorname{err}(p)$ & $\operatorname{err}(\mathbf{u})$ \\
\hline 20 & $1.339 \mathrm{e}-3$ & $3.456 \mathrm{e}-3$ & $2.210 \mathrm{e}-3$ & $4.423 \mathrm{e}-3$ & $1.444 \mathrm{e}-3$ & $4.341 \mathrm{e}-3$ \\
40 & $3.215 \mathrm{e}-4$ & $1.523 \mathrm{e}-3$ & $5.304 \mathrm{e}-4$ & $1.558 \mathrm{e}-3$ & $5.304 \mathrm{e}-4$ & $2.058 \mathrm{e}-3$ \\
80 & $8.416 \mathrm{e}-5$ & $7.690 \mathrm{e}-4$ & $1.359 \mathrm{e}-4$ & $6.841 \mathrm{e}-4$ & $9.014 \mathrm{e}-5$ & $1.027 \mathrm{e}-3$ \\
160 & $2.030 \mathrm{e}-5$ & $3.694 \mathrm{e}-4$ & $3.379 \mathrm{e}-5$ & $2.871 \mathrm{e}-4$ & $2.258 \mathrm{e}-5$ & $5.028 \mathrm{e}-4$ \\
320 & $5.001 \mathrm{e}-6$ & $1.855 \mathrm{e}-4$ & $8.417 \mathrm{e}-6$ & $1.420 \mathrm{e}-4$ & $5.645 \mathrm{e}-6$ & $2.528 \mathrm{e}-4$ \\
\hline rate & 2.01 & 1.05 & 2.00 & 1.24 & 2.06 & 1.02
\end{tabular}

Table 3: Continuous $k$ and sequence of polygonal meshes (Fig. 3, right)

\begin{tabular}{r|cc|cc|cc} 
\# cells & \multicolumn{2}{|c|}{ Scheme I } & \multicolumn{2}{c|}{ Scheme II } & \multicolumn{2}{c}{ Standard MFD } \\
\hline & $\operatorname{err}(p)$ & $\operatorname{err}(\mathbf{u})$ & $\operatorname{err}(p)$ & $\operatorname{err}(\mathbf{u})$ & $\operatorname{err}(p)$ & $\operatorname{err}(\mathbf{u})$ \\
\hline 412 & $8.470 \mathrm{e}-4$ & $6.241 \mathrm{e}-3$ & $2.674 \mathrm{e}-3$ & $3.001 \mathrm{e}-3$ & $1.892 \mathrm{e}-3$ & $4.148 \mathrm{e}-3$ \\
1591 & $2.096 \mathrm{e}-4$ & $2.261 \mathrm{e}-3$ & $6.554 \mathrm{e}-4$ & $9.612 \mathrm{e}-4$ & $4.690 \mathrm{e}-4$ & $1.248 \mathrm{e}-3$ \\
6433 & $5.083 \mathrm{e}-5$ & $8.024 \mathrm{e}-4$ & $1.572 \mathrm{e}-4$ & $4.382 \mathrm{e}-4$ & $1.135 \mathrm{e}-4$ & $4.285 \mathrm{e}-4$ \\
25698 & $1.241 \mathrm{e}-5$ & $2.770 \mathrm{e}-4$ & $3.886 \mathrm{e}-5$ & $1.289 \mathrm{e}-4$ & $2.814 \mathrm{e}-5$ & $1.227 \mathrm{e}-4$ \\
102772 & $3.158 \mathrm{e}-6$ & $1.102 \mathrm{e}-4$ & $9.693 \mathrm{e}-6$ & $5.794 \mathrm{e}-5$ & $6.984 \mathrm{e}-6$ & $5.814 \mathrm{e}-5$ \\
\hline rate & 2.03 & 1.47 & 2.04 & 1.43 & 2.03 & 1.57
\end{tabular}


lack of approximation properties of the discrete inner product (in the space of gradients) when only one velocity degree of freedom is used on a discontinuity face. Scheme III remains to be competitive with the standard MFD scheme [13].

Table 4: Discontinuous $k$ and sequence of smooth meshes (Fig. 3, left)

\begin{tabular}{c|cc|cc|cc}
$1 / h$ & \multicolumn{2}{|c|}{ Scheme I } & \multicolumn{2}{c|}{ Scheme III } & \multicolumn{2}{c}{ Standard MFD } \\
\hline & $\operatorname{err}(p)$ & $\operatorname{err}(\mathbf{u})$ & $\operatorname{err}(p)$ & $\operatorname{err}(\mathbf{u})$ & $\operatorname{err}(p)$ & $\operatorname{err}(\mathbf{u})$ \\
\hline 20 & $1.287 \mathrm{e}-2$ & $6.618 \mathrm{e}-3$ & $1.430 \mathrm{e}-3$ & $3.926 \mathrm{e}-3$ & $1.801 \mathrm{e}-3$ & $5.246 \mathrm{e}-3$ \\
40 & $6.291 \mathrm{e}-3$ & $2.977 \mathrm{e}-3$ & $3.639 \mathrm{e}-4$ & $1.136 \mathrm{e}-4$ & $4.571 \mathrm{e}-4$ & $1.394 \mathrm{e}-3$ \\
80 & $3.127 \mathrm{e}-3$ & $1.584 \mathrm{e}-3$ & $9.137 \mathrm{e}-5$ & $3.308 \mathrm{e}-4$ & $1.147 \mathrm{e}-4$ & $3.616 \mathrm{e}-4$ \\
160 & $1.561 \mathrm{e}-3$ & $9.384 \mathrm{e}-4$ & $2.286 \mathrm{e}-5$ & $9.928 \mathrm{e}-5$ & $2.871 \mathrm{e}-5$ & $9.291 \mathrm{e}-5$ \\
320 & $7.804 \mathrm{e}-4$ & $5.949 \mathrm{e}-4$ & $5.715 \mathrm{e}-6$ & $3.102 \mathrm{e}-5$ & $7.181 \mathrm{e}-6$ & $2.376 \mathrm{e}-5$ \\
\hline rate & 1.01 & 0.86 & 1.99 & 1.42 & 1.99 & 1.95
\end{tabular}

Table 5: Discontinuous $k$ and sequence of randomly perturbed meshes (Fig. 3, center)

\begin{tabular}{c|cc|cc|cc}
$1 / h$ & \multicolumn{2}{|c|}{ Scheme I } & \multicolumn{2}{c|}{ Scheme III } & \multicolumn{2}{c}{ Standard MFD } \\
\hline & $\operatorname{err}(p)$ & $\operatorname{err}(\mathbf{u})$ & $\operatorname{err}(p)$ & $\operatorname{err}(\mathbf{u})$ & $\operatorname{err}(p)$ & $\operatorname{err}(\mathbf{u})$ \\
\hline 20 & $1.239 \mathrm{e}-2$ & $6.798 \mathrm{e}-3$ & $1.446 \mathrm{e}-3$ & $4.541 \mathrm{e}-3$ & $1.290 \mathrm{e}-3$ & $5.320 \mathrm{e}-3$ \\
40 & $6.035 \mathrm{e}-3$ & $3.109 \mathrm{e}-3$ & $3.480 \mathrm{e}-4$ & $1.817 \mathrm{e}-3$ & $3.202 \mathrm{e}-4$ & $2.299 \mathrm{e}-3$ \\
80 & $3.119 \mathrm{e}-3$ & $1.801 \mathrm{e}-3$ & $8.765 \mathrm{e}-5$ & $7.960 \mathrm{e}-4$ & $7.986 \mathrm{e}-5$ & $1.136 \mathrm{e}-3$ \\
160 & $1.485 \mathrm{e}-3$ & $9.869 \mathrm{e}-4$ & $2.216 \mathrm{e}-5$ & $3.598 \mathrm{e}-4$ & $1.998 \mathrm{e}-5$ & $5.544 \mathrm{e}-4$ \\
320 & $7.302 \mathrm{e}-4$ & $6.163 \mathrm{e}-4$ & $5.513 \mathrm{e}-6$ & $1.781 \mathrm{e}-4$ & $5.000 \mathrm{e}-6$ & $2.777 \mathrm{e}-4$ \\
\hline rate & 1.02 & 0.86 & 2.00 & 1.17 & 2.00 & 1.06
\end{tabular}

Table 6: Discontinuous $k$ and sequence of polygonal meshes (Fig. 3, right)

\begin{tabular}{r|cc|cc|cc} 
\# cells & \multicolumn{2}{|c|}{ Scheme I } & \multicolumn{2}{c|}{ Scheme III } & \multicolumn{2}{c}{ Standard MFD } \\
\hline & $\operatorname{err}(p)$ & $\operatorname{err}(\mathbf{u})$ & $\operatorname{err}(p)$ & $\operatorname{err}(\mathbf{u})$ & $\operatorname{err}(p)$ & $\operatorname{err}(\mathbf{u})$ \\
\hline 412 & $4.227 \mathrm{e}-3$ & $7.127 \mathrm{e}-3$ & $2.480 \mathrm{e}-3$ & $4.722 \mathrm{e}-3$ & $2.762 \mathrm{e}-3$ & $7.451 \mathrm{e}-3$ \\
1591 & $2.273 \mathrm{e}-3$ & $2.949 \mathrm{e}-3$ & $6.250 \mathrm{e}-4$ & $1.567 \mathrm{e}-3$ & $6.976 \mathrm{e}-4$ & $2.370 \mathrm{e}-3$ \\
6433 & $1.206 \mathrm{e}-3$ & $1.215 \mathrm{e}-3$ & $1.729 \mathrm{e}-4$ & $6.273 \mathrm{e}-4$ & $1.650 \mathrm{e}-4$ & $9.624 \mathrm{e}-4$ \\
25698 & $5.986 \mathrm{e}-4$ & $6.096 \mathrm{e}-4$ & $3.684 \mathrm{e}-5$ & $1.956 \mathrm{e}-4$ & $4.066 \mathrm{e}-5$ & $2.571 \mathrm{e}-4$ \\
102772 & $3.020 \mathrm{e}-4$ & $3.236 \mathrm{e}-4$ & $9.142 \mathrm{e}-6$ & $8.164 \mathrm{e}-5$ & $1.007 \mathrm{e}-5$ & $1.134 \mathrm{e}-4$ \\
\hline rate & 0.96 & 1.12 & 2.03 & 1.48 & 2.04 & 1.53
\end{tabular}




\subsection{Numerical comparison of MFD schemes in 3D}

In 3D, we observed similar convergence patterns for the schemes. Therefore, in Table 7 we present results only for one set of numerical experiments similar to that in Table 4 . We consider analytic solution given in (4.2), i.e. it is constant in the $z$-direction. The new scheme is competitive with the standard MFD scheme and is slightly more accurate.

Table 7: Discontinuous $k$ and sequence of smooth hexahedral meshes.

\begin{tabular}{c|cc|cc|cc}
$1 / h$ & \multicolumn{2}{|c|}{ Scheme I } & \multicolumn{2}{c|}{ Scheme III } & \multicolumn{2}{c}{ Standard MFD } \\
\hline & $\operatorname{err}(p)$ & $\operatorname{err}(\mathbf{u})$ & $\operatorname{err}(p)$ & $\operatorname{err}(\mathbf{u})$ & $\operatorname{err}(p)$ & $\operatorname{err}(\mathbf{u})$ \\
\hline 10 & $2.394 \mathrm{e}-2$ & $2.558 \mathrm{e}-2$ & $3.930 \mathrm{e}-3$ & $2.291 \mathrm{e}-2$ & $3.987 \mathrm{e}-3$ & $2.547 \mathrm{e}-2$ \\
20 & $1.115 \mathrm{e}-2$ & $9.218 \mathrm{e}-3$ & $1.081 \mathrm{e}-3$ & $6.751 \mathrm{e}-3$ & $1.088 \mathrm{e}-3$ & $7.618 \mathrm{e}-3$ \\
40 & $5.325 \mathrm{e}-3$ & $3.929 \mathrm{e}-3$ & $2.788 \mathrm{e}-4$ & $1.805 \mathrm{e}-3$ & $2.804 \mathrm{e}-4$ & $2.023 \mathrm{e}-3$ \\
80 & $2.601 \mathrm{e}-3$ & $2.048 \mathrm{e}-3$ & $7.026 \mathrm{e}-5$ & $4.816 \mathrm{e}-4$ & $7.071 \mathrm{e}-5$ & $5.197 \mathrm{e}-4$ \\
\hline rate & 1.07 & 1.22 & 1.94 & 1.87 & 1.94 & 1.86
\end{tabular}

\subsection{A parabolic equation}

The above conclusions can be extended to linear parabolic problems with smooth solutions provided that accurate time discretization is employed. But here we consider a specially designed problem with a time-dependent diffusion coefficient that shows importance of the staggered discretization of the diffusion coefficient. The problem was inspired by numerical examples in $[17,14]$. Despite being linear, the solution of this problem has dynamics typical for solutions of nonlinear heat conduction equations.

The backward Euler time discretization of (2.1) reads

$$
\mathbf{u}_{h}^{n+1}=-\mathcal{G} \mathcal{R} \mathcal{A} \mathcal{D} p_{h}^{n+1} \quad \text { and } \quad a \frac{p_{h}^{n+1}-p_{h}^{n}}{\Delta t}+\mathcal{D} \mathcal{I} \mathcal{V}^{k} \mathbf{u}_{h}^{n+1}=\left(b^{n+1}\right)^{I}
$$

where $\Delta t$ is the time step, $p_{h}^{n}$ is the solution at time moment $t^{n}$, and $b^{n+1}=b\left(\mathbf{x}, t^{n+1}\right)$. Inserting the first equation into the second one and using the duality relationship (3.10), we observe that the resulting discrete operator can be symmetrized by multiplying both sides by the diagonal matrix $\mathbb{M}_{\mathcal{P}}$. To reduce the impact of the time integration error, we use small time steps $\Delta t \sim h^{2}$.

Let us consider the parabolic equation $(2.1)$ in rectangular domain $(0,3) \times(0,1)$ with $a=1.0$, zero source term, and the time-dependent diffusion coefficient:

$$
k(\mathbf{x}, t)=\left\{\begin{array}{l}
3 c(c t-x) \text { if } x<c t \\
\epsilon \text { otherwise }
\end{array}\right.
$$

where $c=0.4$. The analytic solution is know for $\epsilon=0$ and is equal to $p^{0}(\mathbf{x}, t)=\sqrt[3]{k}$. This equation approximates a well known Marshak equation for nonlinear heat conduction. 
We set $\epsilon=10^{-9}$ so that we can replace the unknown exact solution $p(\mathbf{x}, t)$ with $p^{0}(\mathbf{x}, t)$ in the error estimates. To be as close as possible to the known solution, we set the timedependent Dirichlet boundary condition $p=p^{0}(0, t)$ on the left side of $\Omega$, the constant boundary condition $p=\sqrt[3]{\epsilon}$ on the right side, and the homogeneous Neumann boundary conditions on the remaining sides. The initial condition is constant equal to $\sqrt[3]{\epsilon}$.

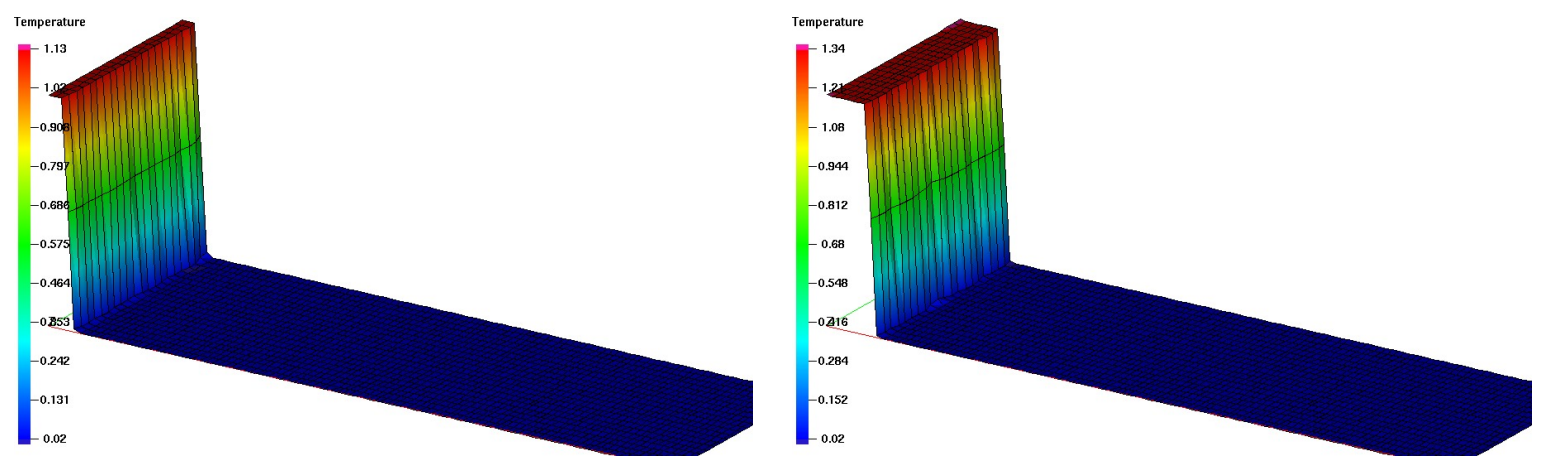

Figure 4: Solution snapshots at times $T=3.0$ and $T=5.0$ for the standard MFD scheme.

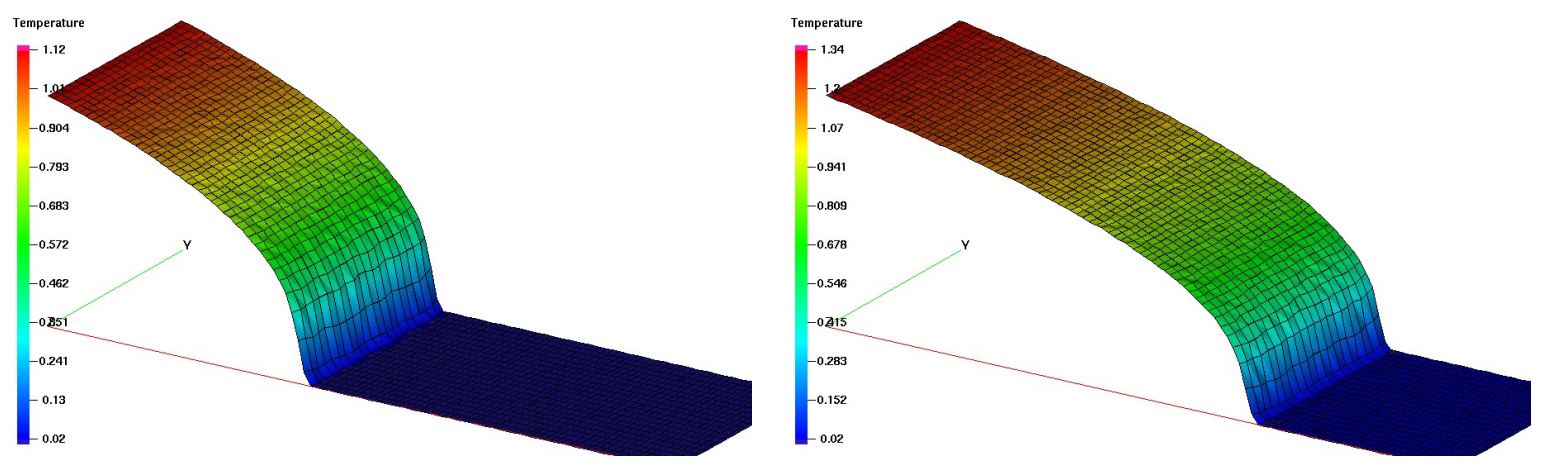

Figure 5: Solution snapshots at times $T=3.0$ and $T=5.0$ for the new MFD scheme.

We solve the parabolic equation on a randomly perturbed quadrilateral mesh that have three times more cells in the x-direction than in the y-direction. The sequence of such meshes is similar to one of the above sequences. We use the arithmetic averaging of the face-based diffusion coefficients $k_{f}$.

For any fixed $t$, function $p^{0}(\mathbf{x}, t)$ is not in $H^{1}(\Omega)$. Although, we do not know regularity of the modified solution $p(\mathbf{x}, t)$, we may expect slower convergence rate on meshes with reasonable resolution. Results of numerical experiments presented in Table 8 confirm this hypothesis. Comparison of Figs 4 and 5 show that the standard MFD scheme leads to incorrect speed of propagation of the solution. 
Table 8: Parabolic equation, spatial error at time $T=3.0$.

\begin{tabular}{c|c|cc} 
Mesh & $\Delta t$ & \multicolumn{2}{|c}{ Scheme III } \\
\hline & & $\operatorname{err}(p)$ & $\operatorname{err}(\mathbf{u})$ \\
\hline $30 \times 10$ & $4.0 \mathrm{e}-3$ & $2.157 \mathrm{e}-2$ & $5.073 \mathrm{e}-2$ \\
$60 \times 20$ & $1.0 \mathrm{e}-3$ & $1.163 \mathrm{e}-2$ & $2.950 \mathrm{e}-2$ \\
$120 \times 40$ & $2.5 \mathrm{e}-4$ & $7.573 \mathrm{e}-3$ & $1.975 \mathrm{e}-2$ \\
\hline rate & & 0.75 & 0.68
\end{tabular}

\section{Conclusion}

Analysis of numerical schemes for nonlinear parabolic equations in one dimension shows that the numerical schemes based on harmonic averaging of cell-centered diffusion coefficients break down when some of these coefficients go to zero or their ratio grows. To address this issue, we developed a novel discrete calculus that leads to a new family of secondorder accurate mimetic finite difference schemes on polygonal and polyhedral meshes. The primary mimetic operator approximates continuum operator $\operatorname{div}(k \cdot)$, the derived (dual) mimetic operator approximates $\nabla(\cdot)$. In the true mimetic spirit, the new mimetic schemes preserve symmetry as well as the positive-definiteness of the continuum problem which allows the use of algebraic solvers with optimal complexity. These schemes use a staggered discretization of the diffusion coefficient, one value per mesh cell and up to two values per mesh face; therefore, they have the flexibility required to build robust numerical algorithms for nonlinear problems. For instances, upwinding of the diffusion coefficients on mesh faces, which is the well established practise for solving nonlinear problems in subsurface flows, can be easily incorporated into the new mimetic schemes. However, the analysis of the new schemes for linear problems is necessary before applying them for solving nonlinear problems. We have shown that the new schemes are competitive with the existing schemes for steady-state problems but provide much more accurate solution for a specially designed transient problem those solution dynamics is similar to that of nonlinear heat conduction problems.

\section{Acknowledgments}

This work was carried out under the auspices of the National Nuclear Security Administration of the U.S. Department of Energy at Los Alamos National Laboratory under Contract No. DE-AC52-06NA25396 and the DOE Office of Science Advanced Scientific Computing Research (ASCR) Program in Applied Mathematics Research.

We are grateful to Rao Garimella (LANL) for helping us with generating dual meshes satisfying geometric constraints. All meshes in this paper were created using his mesh generation toolset MSTK (software.lanl.gov/MeshTools/trac). 


\section{References}

[1] T. Arbogast, C. Dawson, P. Keenan, M. Wheeler, and I. Yotov. Enhanced cell-centered finite differences for elliptic equations on general geometry. SIAM J. Sci. Comput., 19(2):404-425, 1998.

[2] L. Beirao da Veiga, K. Lipnikov, and G. Manzini. The Mimetic Finite Difference Method for Elliptic PDEs. Springer, 2014. 408 pages.

[3] F. Brezzi, K. Lipnikov, and V. Simoncini. A family of mimetic finite difference methods on polygonal and polyhedral meshes. Math. Models Methods Appl. Sci., 15(10):15331551, 2005.

[4] J. Castor. Radiation Hydrodynamics. Cambridge University Press, 2004.

[5] J. Droniou and R. Eymard. A mixed finite volume scheme for anisotropic diffusion problem on any grid. Numer. Math., 1(105):35-71, 2006.

[6] R. Eymard, T. Gallouët, and R. Herbin. The finite volume method. In P. Ciarlet and J. Lions, editors, Handbook for Numerical Analysis, pages 715-1022. North Holland, 2000 .

[7] R. Eymard, T. Gallouët, and R. Herbin. A new finite volume scheme for anisotropic diffusion problems on general grids: convergence analysis. C. R. Math. Acad. Sci. Paris, 344(6):403-406, 2007.

[8] R. Eymard, T. Gallouet, and R. Herbin. Discretization of heterogeneous and anisotropic diffusion problems on general non-conforming meshes. SUSHI: a scheme using stabilization and hybrid interface, 2009. IMA J. Numer. Anal.

[9] B. F., R. Falk, and L. Marini. Basic principle of mixed virtual element methods. ESAIM: Mathematical Modelling and Numerical Analysis, 48(1):1227-1240, 2014.

[10] V. Gyrya, K. Lipnikov, G. Manzini, and D. Svyatskiy. M-adaptation in the mimetic finite difference method. Math. Models Methods Appl. Sci., 24:1621-1663, 2014.

[11] F. Hermeline. A finite volume method for the approximation of diffusion operators on distorted meshes. J. Comput. Phys., 160(2):481-499, 2000.

[12] F. Hermeline. Approximation of diffusion operators with discontinuous tensor coefficients on distorted meshes. Comput. Methods Appl. Mech. Engrg., 192(16-18):1939$1959,2003$.

[13] K. Lipnikov, G. Manzini, and M. Shashkov. Mimetic finite difference method. J. Comp. Phys., 257:1163-1227, 2014.

[14] V. Maslyankin. Convergence of the iterative process for the quasilinear heat transfer equation. USSR Comput. Math. Math. Phys., 17(1):201-210, 1977. 
[15] P. Raviart and J. Thomas. A mixed finite element method for second order elliptic problems. In I. Galligani and E. Magenes, editors, Math. Aspects of Finite Element Method, Lecture Notes in Math. 606. Springer-Verlag, N.Y., 1977.

[16] L. Richards. Capillary conduction of liquids through porous mediums. Physics 1, 5:318-333, 1931.

[17] A. Samarskii and I. Sobol'. Examples of the numerical calculation of temperature waves. USSR Comput. Math. Math. Phys., 3(4):945-970, 1963. 